\title{
Frequency Multiplexing for Deterministic Heralded Single-Photon Sources
}

\author{
Chaitali Joshi, ${ }^{1,2}$ Alessandro Farsi, ${ }^{1}$ Stéphane \\ Clemmen, ${ }^{3}$ Sven Ramelow, ${ }^{4}$ and Alexander L. Gaeta ${ }^{1, *}$ \\ ${ }^{1}$ Department of Applied Physics and Applied Math, \\ Columbia University, New York City, USA \\ ${ }^{2}$ Applied and Engineering Physics, Cornell University, Ithaca, NY, USA \\ ${ }^{3}$ Laboratoire d'Information Quantique, \\ Université Libre de Bruxelles, Bruxelles, Belgium \\ ${ }^{4}$ Institut für Physik, Humboldt-Universität zu Berlin, Berlin, Germany
}

\begin{abstract}
Single-photon sources based on optical parametric processes have been used extensively for quantum information applications due to their flexibility, room-temperature operation and potential for photonic integration. However, the intrinsically probabilistic nature of these sources is a major limitation for realizing large-scale quantum networks. Active feedforward switching of photons from multiple probabilistic sources is a promising approach that can be used to build a deterministic source. However, previous implementations of this approach that utilize spatial and/or temporal multiplexing suffer from rapidly increasing switching losses when scaled to a large number of modes. Here, we break this limitation via frequency multiplexing in which the switching losses remain fixed irrespective of the number of modes. We use the third-order nonlinear process of Bragg scattering four-wave mixing as an efficient ultra-low noise frequency switch and demonstrate multiplexing of three frequency modes. We achieve a record generation rate of $4.6 \times 10^{4}$ multiplexed photons per second with an ultra-low $g^{(2)}(0)=0.07$, indicating high single-photon purity. Our scalable, all-fiber multiplexing system has a total loss of just $1.3 \mathrm{~dB}$ independent of the number of multiplexed modes, such that the $4.8 \mathrm{~dB}$ enhancement from multiplexing three frequency modes markedly overcomes switching loss. Our approach offers a highly promising path to creating a deterministic photon source that can be integrated on a chip-based platform.
\end{abstract}

\footnotetext{
* Corresponding author: alg2207@columbia.edu
} 


\section{INTRODUCTION}

Deterministic and high quality single-photon sources are essential to photonic quantum technologies including communications and information processing. An ideal single-photon source should emit indistinguishable photons in well-defined spatio-temporal and spectral modes with high probability and negligible multi-photon noise. Efforts to build such sources have focused primarily on the following two approaches: sources that rely on nonlinear processes such as spontaneous parametric down conversion (SPDC) or four-wave mixing, and single emitters such as quantum dots, color centers and cavity-coupled atoms and ions [1]. With recent engineering efforts for improved fabrication and control of individual emitters, quantum dots with high brightness and photon purity have been demonstrated [2-4]. However, these sources require cryogenic cooling, lack spectral tunability and are highly sensitive to the host solid-state environment, leading to distinguishability between different emitters $[5]$.

Parametric sources on the other hand can be easily adapted to a wide variety of experimental conditions and have been used for pioneering quantum information experiments including quantum teleportation, loop-hole-free Bell tests and boson sampling [6-10]. These sources operate at room temperature and provide highly indistinguishable photons with flexible control over the spectral and temporal properties of the photons [11-14]. Such sources have proved to be highly versatile, producing photons spanning the visible to the infrared, with bandwidths ranging from a few hundred $\mathrm{kHz}$ to a few $\mathrm{THz}$ [15-17]. Moreover, parametric sources can be fully integrated onto monolithic CMOS-compatible platforms to generate narrow band entangled photons with high brightness [18-20]. However, these sources are fundamentally limited by multi-photon generation, resulting in probabilistic operation with a maximum heralding efficiency of $25 \%$ from a single source.

Active feed-forward switching of photons from multiple identical sources is a promising technique that can overcome the probabilistic operation of a single source [21-25]. By operating individual sources in a regime with low pair production probability, such schemes allow for increasing the single-photon probability without additional multi-photon generation. A key requirement for efficient multiplexing is a low-loss $N \times 1$ switching network that accommodates a sufficiently large number of modes $N$ to achieve deterministic operation. Deterministic operation can be achieved with as few as $N=17$ multiplexed modes with 
a lossless switching network and photon-number resolving (PNR) detectors [26]. Recently, there have been a number of promising demonstrations of multiplexed sources using the spatial and temporal degrees of freedom of a photon [27-33]. However, for both spatial and temporal multiplexing, switching losses increase with the number of modes $N$, which deteriorates enhancement achieved from multiplexing beyond a few modes. Deterministic operation is therefore challenging to achieve without the use of bulky free-space setups [32].

Here, we propose and demonstrate an alternative scheme using frequency multiplexing where losses do not scale with the number of modes. Frequency multiplexing allows for multiple switching operations in a single spatial mode, thus effectively implementing an $N \times 1$ switch in a monolithic optical structure such as a single mode fiber or waveguide. Therefore, distinct from other schemes, switching losses remain fixed irrespective of the number of multiplexed modes $N$. We use tunable quantum frequency translation via Bragg scattering four wave mixing (BS-FWM) [34-37], which we realize with close to unity efficiency and ultra-low noise [38, 39]. Tunable frequency translation allows us to perform active "frequency switching" of multiple frequency channels. We have previously proposed frequency multiplexing and reported preliminary experimental results using two frequency modes [40]. Here, we present a complete characterization of our frequency multiplexing scheme, including theoretical analysis of the scaling performance for large $N$. We present a proof-of-principle demonstration of frequency multiplexing using three frequency modes in an entirely fiberbased setup that leverages on low-loss off the shelf dense wavelength division multiplexing (DWDM) components. With this low-loss and low-noise setup we achieve record generation rates of $46 \mathrm{kHz}$ multiplexed photons with coincidences-to-accidentals ratio exceeding 100 and $g^{(2)}(0)$ of 0.07 . BS-FWM is efficiently tunable over a large bandwidth of more than 1 $\mathrm{THz}$ and therefore our system can be scaled to include a large number of frequency modes, which is required for deterministic photon generation using multiplexing.

We note that recently, multiple research groups have proposed the use of frequency multiplexing as a resource for both continuous variable [41, 42] and circuit-based single-photon QIP applications [43,44]. These proposals emphasize the strong potential of frequency multiplexing for addressing the scaling losses and resource overheads in quantum systems. However, most proposals rely on electro-optic modulators (EOMs) to frequency translate single photons. Recent work [45] discusses a spectrally multiplexed single-photon source using EOMs, but no enhancement in the single-photon rate is demonstrated due to high 
system losses. In addition, EOMs typically have a limited time-bandwidth product close to unity, limiting the maximum frequency shift and the bandwidth of the target pulses. This significantly limits practical implementations to a few frequency modes while exacerbating photon loss due to narrow filtering. Alternatively, our implementation of BS-FWM allows for tunable conversion over $1 \mathrm{THz}$ with an acceptance bandwidth of $100 \mathrm{GHz}$ with few nanosecond pump pulses, which addresses these issues.

\section{PRINCIPLE AND THEORY:}

Figure 1 illustrates our frequency multiplexing scheme. A single source that generates broadband frequency correlated photon pairs is used to create narrowband frequency channels $\left\{\omega_{0}, \omega_{1} \ldots\right\}$. One photon from the pair (heralding photon, not shown) is used to herald the presence of the signal photon. Due to energy conservation, the two photons are correlated in frequency, with the heralding photon providing information about the frequency of the signal photon. This heralding information is used to translate the frequency of the signal photon to the target frequency channel $\omega_{t}$ using tunable frequency conversion. We thus effectively implement an active frequency switch to route photons from multiple frequency bins to a single output frequency channel. In order to be viable as an $N \times 1$ switch for large $N$, the tunable frequency conversion must be efficient over a sufficiently large bandwidth. For this purpose, we use BS-FWM, a third-order nonlinear parametric process involving the coherent interaction between two quantum fields at different frequencies mediated by two strong classical pumps [36]. Contrary to frequency conversion based on parametric amplification, BS-FWM is theoretically noiseless and preserves all quantum properties of the translated photons. BS-FWM allows for independent control of the input and target frequencies by selectively activating auxiliary pumps in the interaction (see Figure 1b). Since phase matching can be achieved by symmetric placement of the classical pumps and quantum fields about the zero-dispersion wavelength of the nonlinear medium, the same setup can be reconfigured to target different frequency shifts by tuning the pump wavelength (see supplementary section I). For efficient conversion, it is critical that the bandwidth of individual channels be less than the acceptance bandwidth $\Delta \nu_{B S}$ of the BS-FWM process for two fixed pumps. This all-optical frequency switch can support ultrafast operation, with the repetition rate limited only to the inverse bandwidth $1 / \Delta \nu_{B S}$. Finally, we note that 
all frequency switching takes place in a single spatial mode (nonlinear fiber/waveguide), as shown in Figure 1c. As additional channels only require additional BS-FWM pumps, no scaling losses are introduced in the path of the single photons.

To understand clearly the characteristics of our frequency multiplexing scheme, we analyze how the performance scales with increasing $N$. Several architectures have been explored for active $N \times 1$ switching of photons in spatial and temporal multiplexing schemes. Typically, these architectures use $2 \times 2$ switches as building blocks for a general $N \times 1$ switch. We compare the performance of the fixed loss scheme with the log-tree network which is generally used for spatial multiplexing and multi-pass binary switches (or storage cavities) generally used in temporal multiplexing [31, 32]. An $N \times 1$ log-tree network has a depth $\left\lceil\log _{2} N\right\rceil$. Assuming a switching efficiency of $\eta_{s}$ per switch, the losses scale as $\eta_{s}^{\left\lceil\log _{2}{ }^{N\rceil}\right.}[46]$. The losses from multi-pass binary switching scale exponentially as $\eta_{s}^{N}$ in the worst case, but we consider an optimized implementation as in Ref. [32]. For our fixed-loss scheme, the switching losses are $\eta_{s}$ irrespective of $N$.

Figure $2 \mathrm{a}$ shows the scaling performance of various schemes. We assume a switching efficiency $\eta_{s}=0.85$ (0.7 dB loss) per switch and all other components, including detectors, are assumed to be ideal. We optimize the emission probability per source $p_{\text {single }}(n=1)$ for each $N$ (see supplementary information section II). The maximum heralding probability for a single source $(N=1)$ is 0.25 . For both log-tree and multi-pass schemes, the single photon probability reaches a maximum of 0.41 and 0.50 , respectively, and saturates due to switching losses for less than $N=10$ multiplexed sources. In contrast, for the fixed-loss scheme, additional multiplexed sources always result in an improvement in the single-photon heralding probability, with a heralding probability of 0.60 for $N=10$ sources. For $N=40$ sources, the fixed-loss scheme achieves $p_{\text {mux }}(n=1)=0.75$, compared with a maximum of 0.89 with a no-loss ideal switching network. Our scheme therefore has an advantage in the intermediate regime of 10 to 20 multiplexed modes as well as asymptotically for large $N$. In order to quantify the effects of practical variability in switching efficiency in implementations of multiplexed sources, we analyze the sensitivity of different schemes to switching losses in Figure 2b. For a moderate increase in losses to $1.2 \mathrm{~dB}$ per switch and 30 multiplexed modes $\left(\eta_{s}=0.75, N=30\right)$, the single photon probability drops significantly from $0.86\left(\eta_{s}=1\right)$ to 0.21, 0.29 for the log-tree and multi-pass schemes, but is reduced only moderately to 0.65 for the fixed-loss scheme. Thus, the frequency multiplexing scheme is significantly more robust 
to switching losses as compared to competing switching architectures in other multiplexed sources.

\section{EXPERIMENTAL SETUP}

We experimentally demonstrate multiplexing of three frequency modes. Figure 3 shows our experimental setup. Our multiplexed source is based on broadband SPDC in a periodically-poled lithium-niobate crystal (PPLN) pumped with a 543-nm CW-laser, generating photon pairs at $940 \mathrm{~nm}$ (heralding photons) and $1280 \mathrm{~nm}$ (heralded signal photons). The heralding photons are sent to a filtering setup consisting of reflecting Bragg gratings (RBG), creating three channels CH0, CH1, CH2 with heralding photons at $\omega_{h, 0}, \omega_{h, 1}, \omega_{h, 2}$, respectively, with 100-GHz bandwidth (see supplementary section III). Each channel is collected into a single-mode fiber and sent to a silicon avalanche single-photon detector, which provides heralding information to the logic circuit. The source crystal temperature is tuned to maximize photon pair production at $\omega_{1}=1280.65 \mathrm{~nm}$ and $\omega_{2}=1280.1 \mathrm{~nm}$, and the pair production at $\omega_{0}=1284.45 \mathrm{~nm}$ is lower by a factor of 0.65 . The heralded signal photons $\left\{\omega_{0}=1284.45 \mathrm{~nm}, \omega_{1}=1280.65 \mathrm{~nm}, \omega_{2}=1280.1 \mathrm{~nm}\right\}$ are injected into the multiplexing setup, comprised of a 100-m nonlinear fiber, wavelength-division-multiplexing couplers and a pump filter (total losses $2.2 \mathrm{~dB}$ ). A single channel centered at $\omega_{t}=1284.45 \mathrm{~nm}$ and 100 $\mathrm{GHz}$ wide, is selected with a tunable grating and then sent to a superconducting nanowire single-photon detector (SNSPD) with a quantum efficiency of $53 \%$.

The nonlinear process of BS-FWM is driven by two pump waves generated by distributed feedback lasers diodes, which determine the frequency shift and hence the input and output frequency channels. The diodes are driven with a 5-ns-long pulsed current source, and the optical pulses (for convenience aligned to the C-band ITU grid) are amplified to a peak level of $10 \mathrm{~W}$ via cascaded erbium-doped fiber amplifiers (EDFA). The pump pulses are combined together, temporally synchronized and aligned in polarization. In order to achieve fast switching operations, we utilize lasers at predetermined wavelengths that are selectively turned on and off via a fast logic circuit controlled by a field programmable gate array (FPGA) (see inset in Figure 3). We measure the conversion efficiency for both process $\omega_{1} \rightarrow \omega_{t}$ and $\omega_{2} \rightarrow \omega_{t}$ to be $93 \%$ (see supplementary section I). 


\begin{tabular}{|c|c|c|c|c|c|}
\hline & $\begin{array}{c}\text { \#Modes } \\
\mathrm{N}\end{array}$ & $\begin{array}{c}\text { Multiplexing } \\
\text { enhancement }\end{array}$ & $\begin{array}{c}\text { Heralding } \\
\text { Efficiency }\end{array}$ & $\begin{array}{c}\text { Single-photon } \\
\text { generation rate }\end{array}$ & $\begin{array}{c}\text { BS-FWM } \\
\text { repetition rate }\end{array}$ \\
\hline $\mathrm{a}$ & 3 & 2.2 & $4.6 \%$ & $46 \mathrm{kHz}$ & $1 \mathrm{MHz}$ \\
\hline $\mathrm{b}$ & 10 & 8.5 & $50 \%$ & $2.5 \mathrm{MHz}$ & $5 \mathrm{MHz}$ \\
\hline
\end{tabular}

Table I. Performance of the frequency multiplexed source a) current system with 3 modes and multiplexing system loss of $1.3 \mathrm{~dB}\left(\eta_{s}=0.75\right)$ b) scaled source with 10 modes and improved system efficiency $\left(\eta_{s}=0.85\right.$, fiber-collection and detection efficiency of $\left.90 \%\right)$

\section{RESULTS}

\section{Heralded single-photon rate}

We first characterize the heralded single-photon rates as functions of SPDC pump power for each individual channel and for the multiplexed source, as shown in Figure 4a. The multiplexed (MUX) source has an enhanced coincidence rate by $4.8 \mathrm{~dB}$ as compared to the mean photon rate of the individual channels. This enhancement significantly overcomes the losses of the setup $(1.3 \mathrm{~dB})$, resulting in a net enhancement of $3.5 \mathrm{~dB}(220 \%)$ in the heralded single-photon rate. At maximum SPDC pump power (25 $\mathrm{mW}$ ), we measure a heralding rate of $1 \mathrm{MHz}$ with a brightness of $23 \mathrm{kHz}$ detected coincidences per second. Supplementary section IV provides detailed characterization of the system efficiency and losses. We estimate a single-photon generation rate of $46 \mathrm{kHz}$ after correcting for detector efficiency $(3 \mathrm{~dB})$, which is the highest reported rate for multiplexed photon sources to date. We note that although simply increasing the pump power of the SPDC source can increase the single-photon generation rate of a single source, this would lead to increased multi-photon generation.

\section{Coincidences-to-accidentals ratio (CAR)}

We measure the coincidences to accidentals ratio (CAR), a standard figure of merit to characterize the multi-photon generation of parametric sources. Figure $4 \mathrm{~b}$ compares the CAR for the multiplexed source and each individual channel. For fair comparison we also measure the coincidence rate and CAR at $\omega_{t}$, directly from the SPDC source, without the 
multiplexing setup in place (referred to as the NoMUX source). We operate in a regime in which the single-photon count rate is much higher than the dark-count rate of the detectors, and therefore the accidental counts are dominated by multi-photon generation, which is inversely proportional to the SPDC pump power. The multiplexed source has a CAR that is a factor of 2 higher throughout as compared to the NoMUX source. For low count rates, the multiplexed source has a CAR exceeding 1000 and remains high at 100 at the maximum count rate. These measurements confirm that the strong classical pumps used in BS-FWM do not introduce significant spurious noise photons even at a high pump trigger rate of 1 $\mathrm{MHz}$.

\section{Single-photon purity $\left(g^{(2)}(0)\right)$}

Finally, we measure the purity of the photons from the multiplexed source by the secondorder correlation function $g^{(2)}=\left\langle\hat{N}_{a} \hat{N}_{b}\right\rangle /\left\langle\hat{N}_{a}\right\rangle\left\langle\hat{N}_{b}\right\rangle$ where $\hat{N}_{a}$ and $\hat{N}_{b}$ are photon number operators corresponding to the two arms of a Hanbury-Brown-Twiss setup [47]. Figure 4c shows the measured $g^{(2)}(0)$ for the multiplexed source and the NoMUX source, for various heralded photon rates. At the maximum heralded photon rate, the multiplexed source has a low $g^{(2)}(0)$ of $0.07 \pm 0.005$. For the same low heralded photon rate of $2.5 \mathrm{kHz}$, the multiplexed source and the NoMUX source have $g^{(2)}(0)$ of $0.015 \pm 0.002$ and $0.056 \pm 0.005$ respectively. The average SPDC pump power required to achieve the same photon rate is a factor of 3 lower for the multiplexed source as compared to the NoMUX source, and therefore has significantly reduced multi-photon generation. The improved single photon purity of the multiplexed source is therefore a strong indicator of successful multiplexing.

The performance of our frequency multiplexed source is comparable with the best multiplexed source demonstrated to-date which implements temporal multiplexing on a free-space optics platform[32]. A complete comparison with other relevant works has been included in the supplementary section $\mathrm{V}$. We achieve a record single-photon generation rate of $46 \mathrm{kHz}$ with an ultra-low $g^{(2)}(0)$ of 0.07 , compared with previously demonstrated $19.3 \mathrm{kHz}$ with a high $g^{(2)}(0)$ of 0.48 [32]. Due to the low loss of our frequency switch $(1.3 \mathrm{~dB})$, we achieve a multiplexing enhancement factor of 2.2 with just three frequency modes. We measure a raw heralding efficiency of $2.3 \%$ and detector-corrected efficiency of $4.6 \%$, which is the highest amongst fiber-based and integrated multiplexed systems demonstrated so far. This 
efficiency is mainly limited by the fiber-collection and spectral filtering loss at the SPDC source and is independent of our "frequency switching" setup. Collection efficiencies as high as $90 \%$ can be achieved by minimizing all transmission and filtering losses, and careful modematching, which would correspond to an order of magnitude improvement in the heralded single-photon rates [10,48]. Another important figure of merit for comparing the different multiplexing implementations is the maximum possible switching speed. In principle, our all-optical frequency switch allows for efficient conversion with repetition rates as high as the inverse of the BS-FWM acceptance bandwidth (100 GHz in this system). Our current implementation can support a repetition rate of $5 \mathrm{MHz}$ and is only limited by the amplification required for the BS-FWM pumps. This amplification requirement can be reduced by increasing the BS-FWM interaction length or using highly nonlinear fibers as the interaction medium, enabling significantly higher repetition rates.

\section{Pulsed operation and scaling}

In order to obtain photons in well-defined temporal modes, pulsed operation is necessary. The efficiency of our "frequency switch" is partially limited to $93 \%$ due to the fluctuations in BS-FWM pump power induced by the randomized trigger arising from $\mathrm{CW}$ operation of the single-photon source. We measure efficiencies as high as $97 \%$ using the same setup with periodic pump triggering. In our existing fiber-based setup, we can incorporate up to 10 frequency modes without a decrease in frequency conversion efficiency. We summarize the scaling performance of our system in Table I. With just 10 multiplexed modes, our system is capable of achieving a single-photon heralding probability exceeding $50 \%$ (per input pump pulse) with a single-photon generation rate of $2.5 \mathrm{MHz}$ (see supplementary section VI). Finally, we note that using cavity-based sources with the spectral line-width of the pump pulse matched to the cavity line-width, it is possible to generate discrete uncorrelated joint

spectral amplitudes [49]. Our system is therefore capable of approaching the regime of deterministic photon generation in pure spectral and temporal modes. 


\section{DISCUSSION}

We have demonstrated a novel frequency multiplexed source with three modes, using highly-efficient low-noise quantum frequency translation. We emphasize that adding additional channels adds complexity only to the BS-FWM pump configuration, and no new components need to be added in the path of the single photons. This ensures that losses remain independent of the number of multiplexed modes. The single spatial mode operation of frequency switching maintains relative polarization stability of photons from different channels from generation to detection, ensuring that the photons are rendered indistinguishable after frequency translation. BS-FWM is fully compatible with the existing optical telecommunication architecture that harnesses dense wavelength division multiplexing (DWDM). The applications of such low-loss high repetition rate frequency multiplexing go beyond single-photon sources and can prove to be highly advantageous for all-photonic quantum repeaters that rely on active feed-forward heralding signals [50]. Our scheme is also entirely adaptable to CMOS-compatible integrated platforms. In particular, integrated comb sources where photons are already confined in well-defined frequency bins can eliminate the need for filtering [18-20] while generating spectrally pure photons [49]. In addition, implementations of BS-FWM in nanophotonic waveguides can significantly reduce pump power and amplification requirements [51]. Frequency multiplexing can thus uniquely harness both fiber and integrated technologies optimized for classical applications to address challenges of scalability in quantum technologies.

\section{Acknowledgements}

This work was funded by the National Science Foundation under Grants PHY-1404300 and EFMA-1641094. S.C. acknowledges the F.R.S.-FNRS for financial support. S.R. acknowledges funding by the DFG (Emmy Noether Program). We thank Aseema Mohanty for useful comments on the manuscript.

\section{Author contributions}

S.C. conceived the idea and performed initial theoretical and experimental groundwork. C.J., A.F. and S.R. implemented the experiment. C.J. and A.F. performed the experiment, 
collected and analyzed the data. All authors contributed to interpreting the data. C.J. and A.F. prepared the manuscript in consultation with all authors. A.G. supervised the project.

[1] Eisaman, M. D., Fan, J., Migdall, A. \& Polyakov, S. V. Invited Review Article: Single-photon sources and detectors. Review of Scientific Instruments 82, 071101 (2011).

[2] Loredo, J. C. et al. Scalable performance in solid-state single-photon sources. Optica 3, 433-440 (2016).

[3] Ding, X. et al. On-demand single photons with high extraction efficiency and near-unity indistinguishability from a resonantly driven quantum dot in a micropillar. Physical Review Letters 116, 020401 (2016).

[4] Somaschi, N. et al. Near-optimal single-photon sources in the solid state. Nature Photonics 10, 340-345 (2016).

[5] Aharonovich, I., Englund, D. \& Toth, M. Solid-state single-photon emitters. Nature Photonics 10, 631-641 (2016).

[6] Ma, X.-S. et al. Quantum teleportation over 143 kilometres using active feed-forward. Nature 489, 269-273 (2012).

[7] Giustina, M. et al. Significant-Loophole-Free Test of Bell's Theorem with Entangled Photons. Physical Review Letters 115, 250401 (2015).

[8] Broome, M. A. et al. Photonic boson sampling in a tunable circuit. Science 339, 794-798 (2013).

[9] Spring, J. B. et al. Boson sampling on a photonic chip. Science 339, 798-801 (2013).

[10] Shalm, L. K. et al. Strong Loophole-Free Test of Local Realism. Physical Review Letters 115, 250402 (2015).

[11] Kwiat, P. G. et al. New high-intensity source of polarization-entangled photon pairs. Physical Review Letters 75, 4337 (1995).

[12] Kwiat, P. G., Waks, E., White, A. G., Appelbaum, I. \& Eberhard, P. H. Ultrabright source of polarization-entangled photons. Physical Review A 60, R773 (1999).

[13] Tanzilli, S. et al. Highly efficient photon-pair source using periodically poled lithium niobate waveguide. Electronics Letters 37, 26 (2001). 
[14] Fedrizzi, A., Herbst, T., Poppe, A., Jennewein, T. \& Zeilinger, A. A wavelength-tunable fibercoupled source of narrowband entangled photons. Optics Express 15, 15377-15386 (2007).

[15] Rambach, M., Nikolova, A., Weinhold, T. J. \& White, A. G. Sub-megahertz linewidth single photon source. APL Photonics 1, 096101 (2016).

[16] Nasr, M. B. et al. Ultrabroadband Biphotons Generated via Chirped Quasi-Phase-Matched Optical Parametric Down-Conversion. Physical Review Letters 100, 183601 (2008).

[17] O'Donnell, K. A. \& U'Ren, A. B. Observation of ultrabroadband, beamlike parametric downconversion. Optics Letters 32, 817-819 (2007).

[18] Reimer, C. et al. Integrated frequency comb source of heralded single photons. Optics Express 22, 6535 (2014).

[19] Reimer, C. et al. Generation of multiphoton entangled quantum states by means of integrated frequency combs. Science 351, 1176-1180 (2016).

[20] Ramelow, S. et al. Silicon-Nitride Platform for Narrowband Entangled Photon Generation. arXiv:1508.04358 [physics, physics:quant-ph] (2015). 1508.04358.

[21] Migdall, A. L., Branning, D. \& Castelletto, S. Tailoring single-photon and multiphoton probabilities of a single-photon on-demand source. Physical Review A 66, 053805 (2002).

[22] Jeffrey, E., Peters, N. A. \& Kwiat, P. G. Towards a periodic deterministic source of arbitrary single-photon states. New Journal of Physics 6, 100-100 (2004).

[23] Pittman, T. B., Jacobs, B. C. \& Franson, J. D. Single photons on pseudodemand from stored parametric down-conversion. Physical Review A 66, 042303 (2002).

[24] Shapiro, J. H. \& Wong, F. N. On-demand single-photon generation using a modular array of parametric downconverters with electro-optic polarization controls. Optics Letters 32, 26982700 (2007).

[25] Mower, J. \& Englund, D. Efficient generation of single and entangled photons on a silicon photonic integrated chip. Physical Review A 84, 052326 (2011).

[26] Christ, A. \& Silberhorn, C. Limits on the deterministic creation of pure single-photon states using parametric down-conversion. Physical Review A 85, 023829 (2012).

[27] Ma, X.-s., Zotter, S., Kofler, J., Jennewein, T. \& Zeilinger, A. Experimental generation of single photons via active multiplexing. Physical Review A 83, 043814 (2011).

[28] Broome, M. A., Almeida, M. P., Fedrizzi, A. \& White, A. G. Reducing multi-photon rates in pulsed down-conversion by temporal multiplexing. Optics Express 19, 22698 (2011). 
[29] Collins, M. J. et al. Integrated spatial multiplexing of heralded single-photon sources. Nature communications 4, 2582 (2013).

[30] Mendoza, G. J. et al. Active temporal and spatial multiplexing of photons. Optica 3, 127 (2016).

[31] Xiong, C. et al. Active temporal multiplexing of indistinguishable heralded single photons. Nature Communications 7, 10853 (2016).

[32] Kaneda, F. et al. Time-multiplexed heralded single-photon source. Optica 2, 1010-1013 (2015).

[33] Francis-Jones, R. J. A., Hoggarth, R. A. \& Mosley, P. J. All-fiber multiplexed source of high-purity single photons. Optica 3, 1270-1273 (2016).

[34] Inoue, K. Tunable and selective wavelength conversion using fiber four-wave mixing with two pump lights. IEEE Photonics Technology Letters 6, 1451-1453 (1994).

[35] McKinstrie, C. J., Harvey, J. D., Radic, S. \& Raymer, M. G. Translation of quantum states by four-wave mixing in fibers. Optics Express 13, 9131 (2005).

[36] McGuinness, H. J., Raymer, M. G., McKinstrie, C. J. \& Radic, S. Quantum Frequency Translation of Single-Photon States in a Photonic Crystal Fiber. Physical Review Letters 105, $093604(2010)$.

[37] Li, Q., Davanço, M. \& Srinivasan, K. Efficient and low-noise single-photon-level frequency conversion interfaces using silicon nanophotonics. Nature Photonics 10, 406-414 (2016).

[38] Farsi, A., Clemmen, S., Ramelow, S. \& Gaeta, A. L. Low-noise quantum frequency translation of single photons. FM3A.4 (OSA, 2015).

[39] Clemmen, S., Farsi, A., Ramelow, S. \& Gaeta, A. L. Ramsey interference with single photons. Physical Review Letters 117, 223601 (2016).

[40] Joshi, C., Farsi, A., Ramelow, S., Clemmen, S. \& Gaeta, A. L. Frequency multiplexing for quasi-deterministic heralded single-photon sources. In Conference on Lasers and Electro-Optics (2016), paper FTh1C.2, FTh1C.2 (Optical Society of America, 2016).

[41] Roslund, J., de Araújo, R. M., Jiang, S., Fabre, C. \& Treps, N. Wavelength-multiplexed quantum networks with ultrafast frequency combs. Nature Photonics 8, 109-112 (2014).

[42] Humphreys, P. C. et al. Continuous-variable quantum computing in optical time-frequency modes using quantum memories. Physical Review Letters 113, 130502 (2014).

[43] Sinclair, N. et al. Spectral Multiplexing for Scalable Quantum Photonics using an Atomic Frequency Comb Quantum Memory and Feed-Forward Control. Physical Review Letters 113, 
053603 (2014).

[44] Lukens, J. M. \& Lougovski, P. Frequency-encoded photonic qubits for scalable quantum information processing. Optica 4, 8-16 (2017).

[45] Puigibert, M. G. et al. Heralded single photons based on spectral multiplexing and feed-forward control. arXiv:1703.02068 [quant-ph] (2017). 1703.02068.

[46] Bonneau, D., Mendoza, G. J., O’Brien, J. L. \& Thompson, M. G. Effect of loss on multiplexed single-photon sources. New Journal of Physics 17, 043057 (2015).

[47] Brown, R. H. \& Twiss, R. Q. Correlation between Photons in two Coherent Beams of Light. Nature 177, 27-29 (1956).

[48] Ramelow, S. et al. Highly efficient heralding of entangled single photons. Optics Express 21, 6707 (2013).

[49] Helt, L. G., Yang, Z., Liscidini, M. \& Sipe, J. E. Spontaneous four-wave mixing in microring resonators. Optics Letters 35, 3006-3008 (2010).

[50] Azuma, K., Tamaki, K. \& Lo, H.-K. All-photonic quantum repeaters. Nature Communications 6, ncomms7787 (2015).

[51] Agha, I., Davanço, M., Thurston, B. \& Srinivasan, K. Low-noise chip-based frequency conversion by four-wave-mixing Bragg scattering in $\mathrm{SiN}_{\mathrm{x}}$ waveguides. Optics Letters 37, 2997-2999 (2012). 


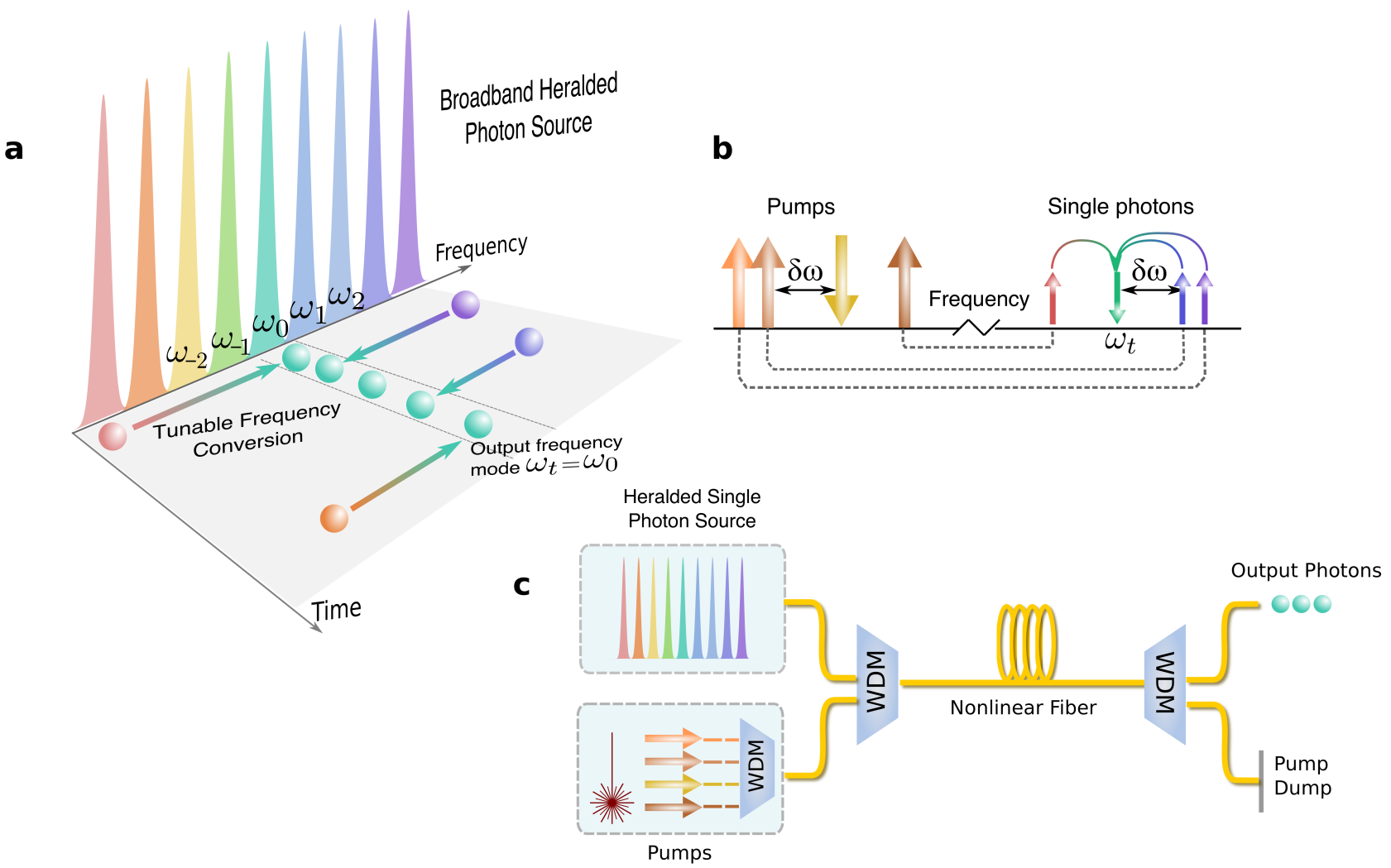

Figure 1. Principle of a frequency multiplexed single-photon source. a) Multiple narrowband frequency channels $\left\{\omega_{0}, \omega_{1} \ldots\right\}$ are extracted from a broadband single-photon source. Tunable frequency conversion is used to convert photons from different channels to a common target frequency mode $\omega_{t}$. b) Tunable frequency conversion using Bragg scattering four-wave mixing (BS-FWM): Two strong classical pumps drive the interaction between the input and target $\left(\omega_{t}\right)$ single-photon fields. The frequency separation $\delta \omega$ between the pump fields determines the frequency shift of the single photons, and additional pump fields can be used to increase the number of possible values of $\delta \omega . \mathbf{c})$ Fixed-loss operation of frequency multiplexing: single photons and BSFWM pumps are combined using wavelength division multiplexers (WDM), and all active frequency switching takes place in a single nonlinear fiber/waveguide. Additional channels can be added by introducing additional pumps, without introducing losses in the path of the single photons. 


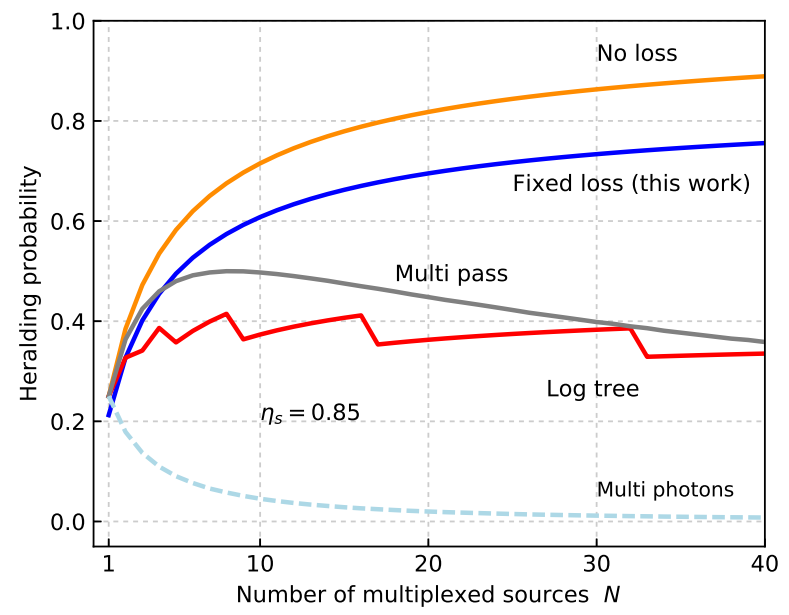

(a)

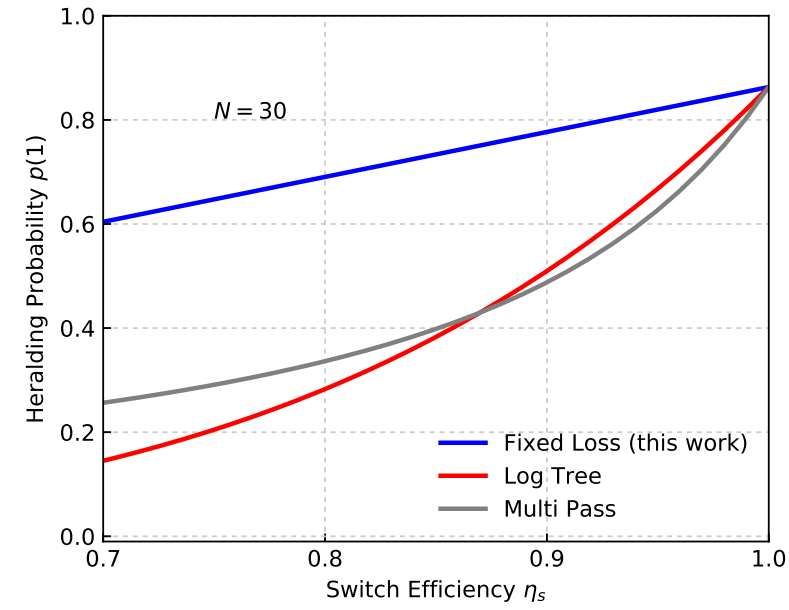

(b)

Figure 2. Theoretical prediction of scaling performance for various switching schemes.

a) The maximum single-photon heralding probability for a single source $(N=1)$ is 0.25 . For an efficiency of $\eta_{s}=0.85$ ( $0.7 \mathrm{~dB}$ loss) per switch, the single-photon emission probability for both log-tree and multi-pass schemes reaches a maximum of $0.41,0.50$, respectively, and then saturates for large $N$ due to scaling losses. In contrast, additional multiplexed sources always result in improved performance for the fixed-loss scheme, with $p_{m u x}(n=1)=0.75$ for $N=40$ sources. The multi-photon emission probability $p_{m u x}(n>1)$, ignoring switching losses, is shown as the dashed light blue curve, and is less than $1 \%$ for $N=40$ modes. b) Single-photon heralding probability for various switching schemes as a function of switch loss, for a fixed number of sources $N=30$. The fixed-loss scheme is significantly more robust to variability in switching losses. Note that the maximum heralding probability for $\eta_{s}=1$ is not equal to 1 since the heralding detectors are non-photon-number resolving. 


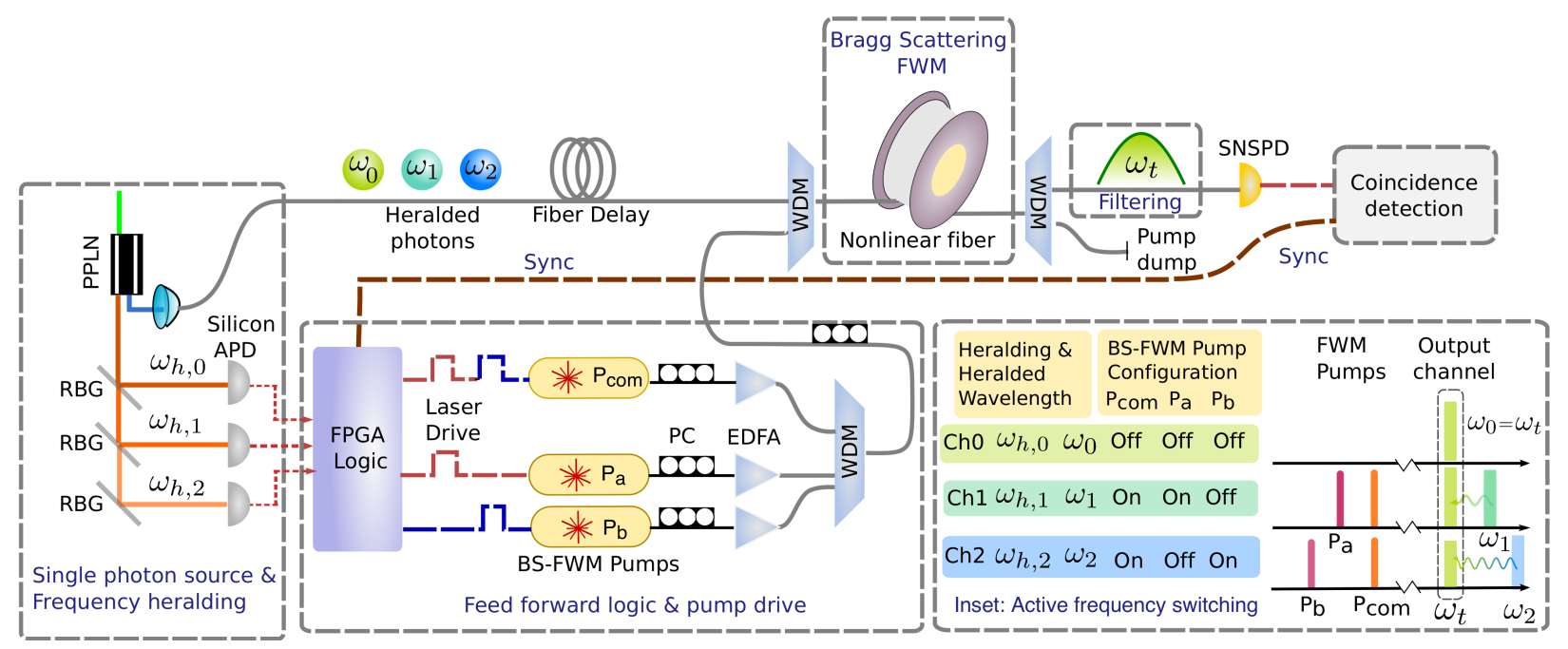

Figure 3. Experimental setup for multiplexing of three frequency modes. PPLN - periodically poled lithium niobate, RBG - reflecting Bragg grating, FPGA - Field programmable gate array, PC: polarization control, EDFA: erbium-doped fiber amplifier, WDM- wavelength division multiplexer, BS-FWM - Bragg scattering four-wave mixing, SNSPD: superconducting nanowire single-photon detectors. A PPLN crystal is pumped with a CW laser at $543 \mathrm{~nm}$, generating photon pairs at $940 \mathrm{~nm}$ (heralding photons) and $1280 \mathrm{~nm}$ (heralded photons). The heralding photons are filtered into three channels $\left\{\omega_{h, 0}, \omega_{h, 1}, \omega_{h, 2}\right\}$ with $100 \mathrm{GHz}$ bandwidth, with corresponding heralded signal photons as $\left\{\omega_{0}, \omega_{1}, \omega_{2}\right\}$. An FPGA is used to process this heralding information and selectively activate the BS-FWM pumps (see inset), such that the heralded photons are switched to the target frequency $\omega_{t}$. The signal photons are combined with the BS-FWM pumps using WDMs, and sent to the nonlinear fiber. A free-space filtering setup extracts photons at $\omega_{t}$ at the output, which are then sent to an SNSPD. A time-tagging module is used for coincidence measurements between the FPGA processed heralding trigger (sync) and the output of the SNSPD. 


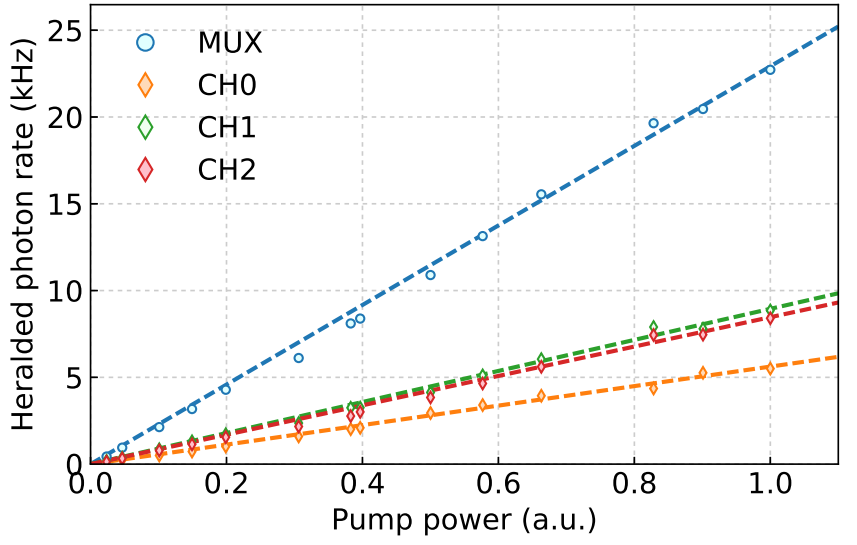

(a)

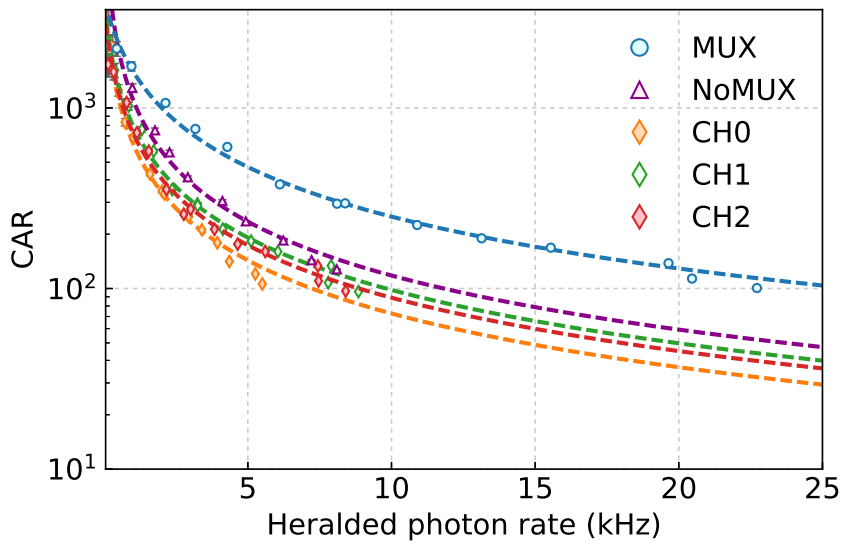

(b)

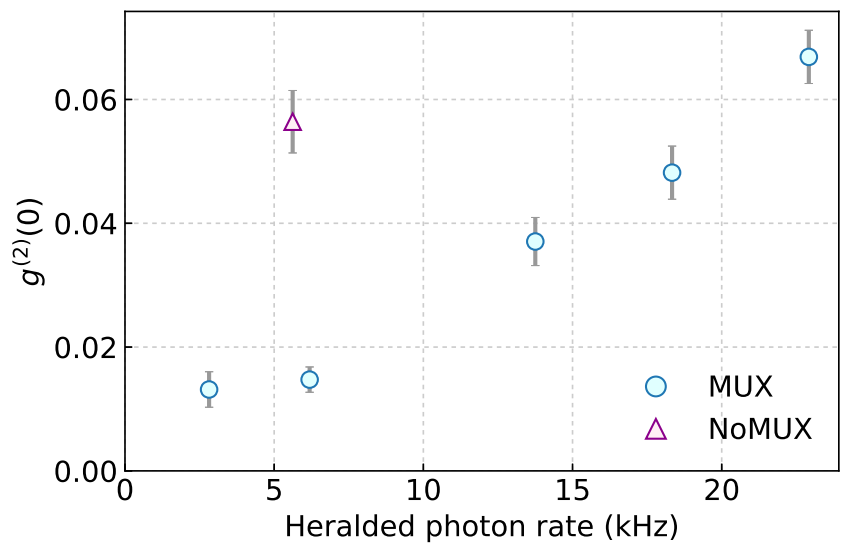

(c)

Figure 4. Experimental results from multiplexing of three frequency modes. Blue circles: multiplexed (MUX) source, purple triangles: NoMUX source, diamonds: contributions from the individual channels (orange - $\mathrm{CH} 0$, green - $\mathrm{CH} 1$, red - CH2). a) Total coincidence counts (heralded photons) as a function of SPDC pump power: The multiplexed source has an enhanced coincidence rate by $4.8 \mathrm{~dB}$ as compared to the mean of the individual channels and overcomes the losses of the setup (1.3 dB), with a net enhancement of $220 \%$. At maximum SPDC pump power (25 $\mathrm{mW})$, we measure a heralded photon rate of $23 \mathrm{kHz}$ from the multiplexed source. b) Coincidences to accidentals ratio (CAR) vs coincidences: For a fixed coincidence rate, the multiplexed source has a CAR that is a factor of 2 higher as compared to the NoMUX source. For low coincidence rates, the multiplexed source has a CAR exceeding 1000 and remains high at 100 for large coincidence rates. c) Measurement of $g^{(2)}(0)$ : The multiplexed source has a low $g^{(2)}(0)$ of $0.07 \pm 0.005$ for large coincidence rates. For the same coincidence rate of $2.5 \mathrm{kHz}$ the multiplexed source has an improved single-photon purity with $g^{(2)}(0)$ of $0.015 \pm 0.002$ as compared to the NoMUX source with a $g^{(2)}(0)$ of $0.056 \pm 0.005$. Error bars are estimated using Poisson statistics. 


\section{Supplementary Information}

\section{BRAGG SCATTERING FOUR-WAVE MIXING}

Four wave Mixing Bragg scattering is a parametric process driven by two strong pump fields at $\omega_{P 1}$ and $\omega_{P 2}$ separated by $\Delta \omega=\omega_{P 1}-\omega_{P 2}$ that can convert an input field $\omega_{i}$ to $\omega_{t}=\omega_{i}-\Delta \omega$ (see Figure S1a). The efficiency of the conversion is $\eta=\frac{k^{2}}{k^{2}+\kappa^{2}} \sin \left(\sqrt{k^{2}+\kappa^{2}} L\right)$ where $\kappa=2 P \gamma$ is the nonlinear strength, $k=\frac{1}{2}\left(\beta_{P 1}-\beta_{P 2}+\beta_{t}-\beta_{i}\right)$ is the phase mismatch, $L$ the interaction length, $P$ is the power in each pump, $\gamma$ is the nonlinear coefficient of the medium, and $\beta_{P 1, P 2, t, i}$ the propagation vector for each participating fields. At the end of the interaction, the signal intensity is depleted by a factor of $1-\eta$. For a perfectly phase-matched process $(k=0)$, complete conversion is achieved when the interaction strength $2 \gamma P L$ equals $\pi / 2$ (figure S1b). Tunable frequency conversion can be achieved by tuning the separation $\Delta \omega$ between the two strong pumps. Phase matching is achieved by symmetric placement of the pumps and the input and target fields around the zero-dispersion wavelength $\left(\beta^{(2)}=0\right.$ ). The acceptance bandwidth $\Delta \nu_{B S}$ of this process is determined by higher order dispersion.

The setup used for this experiment is similar to [38, 39]. We use a 100-m long dispersion shifted fiber with the zero dispersion wavelength $\lambda_{z g v d}=1405 \mathrm{~nm}$ as the nonlinear medium. The fiber is cooled using liquid nitrogen in order to remove spontaneous Raman noise. Pumps are generated by temperature stabilized distributed feed-back laser diodes and combined together using DWDMs. Within the bandwidth of the filter on the target channel, we measure a background of $3 \times 10^{-3}$ photons per pump pulse duration. We achieve $93 \%$ conversion efficiency with this setup, as shown in S1c. The conversion efficiency is limited by the fluctuations in the pump power due to random triggering set by the CW nature of the photon source. With a fixed periodic trigger rate, we measure a conversion rate of $97 \%$ . Our filtering bandwidth at the target output is set to $100 \mathrm{GHz}$ (figure S1e), which is less than the acceptance bandwidth of the process $\left(\Delta \nu_{B S}=160 \mathrm{GHz}\right)$. 


\begin{tabular}{|c|c|c|c|c|c|c|c|c|}
\hline Reference & $\begin{array}{c}\text { Multiplexing } \\
\text { scheme }\end{array}$ & Platform & $\begin{array}{c}\text { \#Modes } \\
\mathrm{N}\end{array}$ & $\begin{array}{c}\text { Single- } \\
\text { photon } \\
\text { detection } \\
\text { rate }(\mathrm{kHz})\end{array}$ & $\begin{array}{c}\text { Enhancement } \\
\text { factor }\end{array}$ & $g^{(2)}(0)$ & $\begin{array}{l}\text { Heralding } \\
\text { efficiency }\end{array}$ & $\begin{array}{c}\text { Max. } \\
\text { switching } \\
\text { speed }\end{array}$ \\
\hline This work & Frequency & Fiber & 3 & $23\left(46^{\dagger}\right)$ & 2.2 & 0.07 & $\begin{array}{c}2.3 \% \\
\left(4.6 \%{ }^{\dagger}\right)\end{array}$ & $5 \mathrm{MHz}$ \\
\hline $\begin{array}{c}\text { Kaneda et al. } \\
\text { [32] }\end{array}$ & Time & Free space & 30 & $11\left(19.3^{\dagger}\right)$ & 6 & 0.48 & $\begin{array}{c}22 \% \\
\left(38.6 \%{ }^{\dagger}\right)\end{array}$ & $50 \mathrm{kHz}$ \\
\hline $\begin{array}{c}\text { Xiong et al. } \\
{[31]}\end{array}$ & Time & Fiber & 4 & 0.6 & 2 & $\ldots$ & $\ldots$ & $\ldots$ \\
\hline $\begin{array}{c}\text { Mendoza et } \\
\text { al. [30] }\end{array}$ & Space-Time & Fiber & 8 & 0.4 & 1.8 & $\ldots$ & $\leq 0.5 \%^{*}$ & $500 \mathrm{kHz}$ \\
\hline $\begin{array}{c}\text { Puigibert et } \\
\text { al.[45] }\end{array}$ & Frequency & Fiber & 3 & 0.4 & 1 & 0.06 & $\ldots$ & $\ldots$ \\
\hline $\begin{array}{c}\text { Francis-Jones } \\
\text { et al.[33] }\end{array}$ & Space & Fiber & 2 & 0.6 & 1.4 & 0.05 & $\ldots$ & $\ldots$ \\
\hline Ma et al.[27] & Space & Free space & 4 & 0.7 & 1.4 & 0.1 & $\ldots$ & $15 \mathrm{MHz}$ \\
\hline $\begin{array}{l}\text { Collins et al. } \\
\qquad[29]\end{array}$ & Space & Integrated & 2 & 0.02 & 1.6 & 0.2 & $1 \%$ & $1 \mathrm{MHz}$ \\
\hline
\end{tabular}

${ }^{\dagger}$ generation rates are estimated after correcting for detection loss

*estimated from reported data

Table S1. Comparison of the performance of the frequency multiplexed source with other demonstrations of multiplexed single photon sources using various schemes and platforms.

\section{THEORY OF MULTIPLEXED SOURCES}

We adapt the analysis presented in References $[26,46]$. We assume that the SPDC source generates a two-mode squeezed state:

$$
|\psi\rangle=\sqrt{1-|\xi|^{2}} \sum_{n=0}^{n=\infty} \xi^{n}\left|n_{s}, n_{i}\right\rangle
$$


where $\xi$ is the squeezing parameter.

Assuming the use of bucket (non-photon number resolving detectors), the total heralding probability is obtained by summing over the probability of an n-photon fock state registering a 'click' on the detector, given by $\left[1-\left(1-\eta_{h}\right)^{n}\right]$, over all $\mathrm{n}$.

$$
p_{h}=\frac{\eta_{h}|\xi|^{2}}{1-\left(1-\eta_{h}\right)|\xi|^{2}}
$$

where $\eta_{h}$ is the net effciency on the heralding arm.

The conditional probability of detecting a single photon on the heralded arm is then given by:

$$
p_{s}=\frac{1}{p_{h}} \eta_{d} \eta_{h}|\xi|^{2}\left(1-|\xi|^{2}\right)
$$

where $\eta_{d}$ is the net efficiency on the heralded photon arm. For $N$ multiplexed sources, the probability that a heralding photon is registered in at least one of the $N$ sources is given as:

$$
p_{h}^{\operatorname{mux}}(N)=1-\left(1-p_{h}\right)^{N}
$$

If the net efficiency of the switching network is $\eta_{\text {switch }}$, the corresponding total probability that a single photon is heralded at the ouput is given by combining Eqns. 4 and 5:

$$
p_{s}^{m u x}(N)=\eta_{\text {switch }} \times p_{s} \times p_{h}^{m u x}(N)
$$

Here the loss factor $\eta_{\text {switch }}$ for the fixed loss and log tree architectures is given as:

$$
\begin{aligned}
\eta_{\text {switch }}^{\text {fixed-loss }} & =\eta_{s} \\
\eta_{\text {switch }}^{\text {log-tree }} & =\eta_{s}^{\left\lceil\log _{2} N\right\rceil}
\end{aligned}
$$

where $\eta_{s}$ is the switching effciency per switch. The expressions for the multi-pass scheme are slightly more involved. We assume optimization similar to that in Ref. [32] where losses are minimized by routing photons from the the last heralded slot in case of temporal multiplexing. The total heralding probability in this case is given by:

$$
p_{s}^{m u x}=\sum_{j=1}^{N}\left(1-p_{h}\right)^{N-j} p_{h} \times p_{s} \eta_{s}^{N-j}
$$

In Eqn [8] the first term is the probability that no photon was heralded in the last $N-j$ slots,and the corresponding switching losses are equal to $\eta_{s}^{N-j}$. We note that we do not 
consider the cases where multi-photon emission is detected as a single photon event due to switching losses. In order to isolate effects of switching losses, we assume all other components including detection to be perfect $\left(\eta_{d}=1, \eta_{h}=1\right)$.

While determining the scaling performance of the multiplexed sources for various $N$, we optimize the mean photon number (or equivalently the squeezing parameter) for each $N$. The squeezing parameter $\xi$ is related to the mean photon number in the signal and idler modes as:

$$
\mu=\frac{|\xi|^{2}}{1-|\xi|^{2}}
$$

For small heralding probability $p_{h}$, the probability that atleast one multiplexed source triggers is $N p_{h}$. As $N$ increases, optimal performance is achieved for lower mean photon number $\mu$, as shown in Fig. S2. Therefore, to obtain the scaling performance of the schemes we optimize $\mu$ for each $N$, maintaining this $\mu$ across schemes.

Finally, the conditional multi-photon probability for a given squeezing parameter is (ignoring switching losses):

$$
\begin{aligned}
p_{\text {multi }} & =|\xi|^{4} \\
& =\left(\frac{\mu}{1+\mu}\right)^{2}
\end{aligned}
$$

As the mean photon number is reduced for increasing $N$, the multi-photon noise correspondingly reduces for large $N$.

\section{SOURCE CHARACTERIZATION}

We characterize the spectrum of our idler (heralding) photons using a single photon spectrometer (Ocean Optics). The results are shown in Fig. S3. The heralding photons are

filtered into $100 \mathrm{GHz}$ wide channels using reflecting Bragg gratings. The highlighted regions reflect the corresponding heralded channels.

\section{CHARACTERIZATION OF SYSTEM EFFICIENCY}

We measure the heralding efficiency on the multiplexed photon arm by measuring the ratio of the detected coincidences to the heralding rate, as shown in Fig. S4. The raw 
heralding efficiency is about $2.3 \%$. Without the multiplexing setup in place, we measure a heralding efficiency of $3 \%$, corresponding to a $1.3 \mathrm{~dB}$ loss due to the multiplexing BSFWM setup. The losses in the path of the multiplexed photon after collection from the SPDC source were measured to be: 1.3 dB BS-FWM setup (WDMs and nonlinear fiber), 1 $\mathrm{dB}$ free-space filtering grating, $2.5 \mathrm{~dB}$ fiber-coupling after filtering and $3 \mathrm{~dB}$ detection loss. After accounting for detection loss, we infer a heralding probability of $4.6 \%$. We estimate about $8 \mathrm{~dB}$ losses at collection from the SPDC source, primarily due to mode-mismatch and transmission loss from filtering optics.

\section{COMPARISON WITH PREVIOUS DEMONSTRATIONS OF MULTIPLEXED PHOTON SOURCES}

In Table S1, we compare the the performance characteristics of our frequency multiplexed source with previous demonstrations of multiplexing using different schemes and platforms. Generated rates are calculated after accounting for detector inefficiency. We note that the maximum switching speed of our system is only limited by the amplification required for the BS-FWM pumps. In principle, our system can be operated at a repetition rate of $1 / \Delta \nu_{B S}$ where $\Delta \nu_{B S}$ is the acceptance bandwidth of BS-FWM, which is $100 \mathrm{GHz}$ for our current implementation.

\section{FREQUENCY MULTIPLEXING SCALING}

Here we show how an implementation of frequency multiplexing using BS-FWM can support large number of frequency channels without drop in conversion efficiency and achieve a 50\% single-photon heralding probability with just 10 multiplexed modes.

We introduce for convenience average frequency $\Delta \Omega=\left(\omega_{P 1}+\omega_{P 2}\right) / 2-\omega_{Z D W}$ and of the frequency offset $\widetilde{\omega}=\omega_{i}-\left(\Delta \omega / 2+\Delta \Omega+\omega_{Z D W}\right)$, where $\Delta \omega=\omega_{P 1}-\omega_{P 2}$ is the separation between the pumps (see S1a). The phase mismatch $k=\beta\left(\omega_{t}\right)-\beta\left(\omega_{i}\right)-\beta\left(\omega_{P 1}\right)+\beta\left(\omega_{P 2}\right)$ for a BS-FWM can then be expanded around the zero dispersion frequency $\omega_{Z D W}$ as follows: 


$$
\begin{gathered}
k=\frac{\beta^{(3)}}{6}\left[\left(\Delta \Omega+\tilde{\omega}-\frac{\Delta \omega}{2}\right)^{3}-\left(\Delta \Omega+\tilde{\omega}+\frac{\Delta \omega}{2}\right)^{3}\right. \\
\left.-\left(-\Delta \Omega-\frac{\Delta \omega}{2}\right)^{3}+\left(-\Delta \Omega+\frac{\Delta \omega}{2}\right)^{3}\right]+\mathcal{O}\left(\beta^{(4)}\right) \\
k=\frac{\beta^{(3)}}{6}[3 \tilde{\omega} \Delta \omega(\tilde{\omega}+2 \Delta \Omega)]
\end{gathered}
$$

This shows that shows that phase-matching can always be fulfilled by choosing $\widetilde{\omega}=0$. While the condition $k=0$ is always satisfied, for large detuning $\Delta \omega$ between the input and the target frequency $\omega_{t}$, the acceptance bandwidth is modified and depends on the detuning $\Delta \omega$. The target bandwidth must match the acceptance bandwidth for optimal conversion.

In Figure S5a we show that we can add up to 10 additional channels separated by $100 \mathrm{GHz}$ without significant reduction in the conversion efficiency. The phase-mismatch $k$ is calculated for the dispersion profile of the nonlinear fiber used in our experiment, shown in Figure S1d. Here, we assume a fixed target frequency $\omega_{t}$, and we fix one pump while detuning the other such that the frequency separation between the pumps equals the frequency separation between the input and target. The maximum conversion efficiency is maintained for all 10 channels. The acceptance bandwidth however reduces by a factor of 2 , from $160 \mathrm{GHz}$ for the first channel to $70 \mathrm{GHz}$ for the 10th channel. Additional channels will therefore require stronger filtering.

Figure S5b shows the calculated scaling performance of the frequency multiplexed source for 10 modes. We assume a feasible value for detector and fiber-collection efficiency of $90 \%$. Heralding efficiencies as high as 50\% can be achieved using just 10 multiplexed modes. 

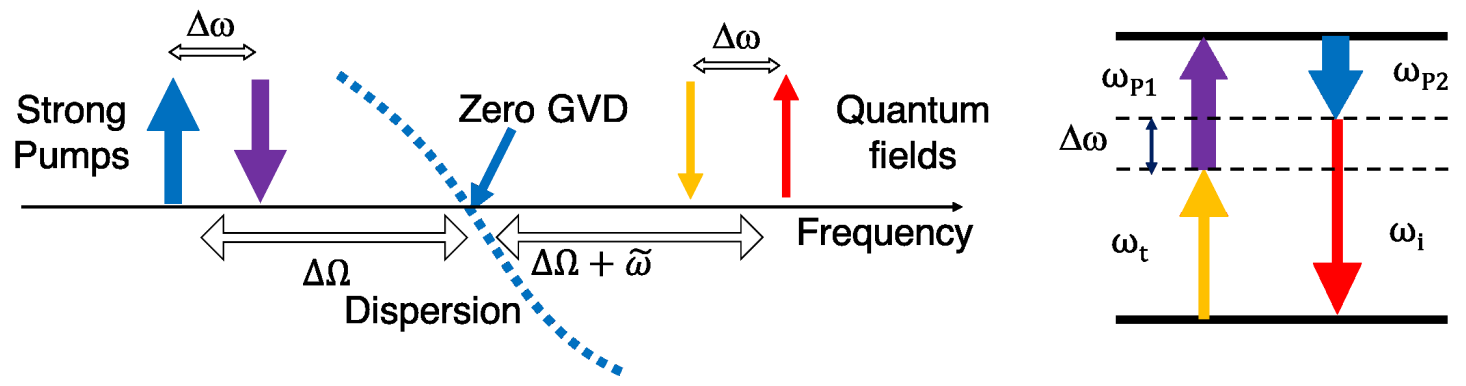

(a)

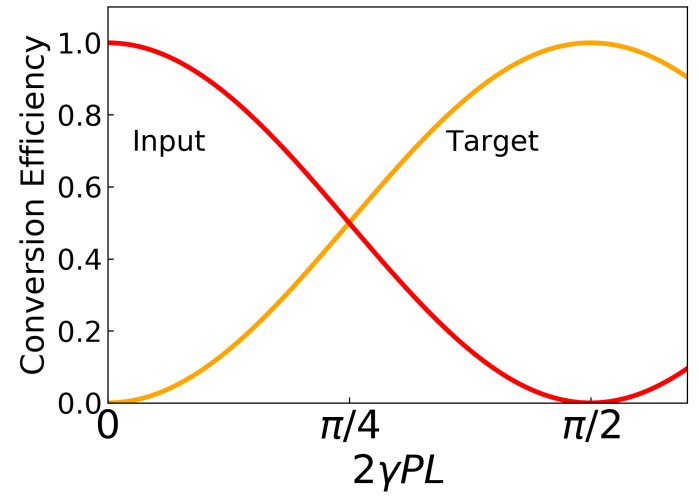

(b)

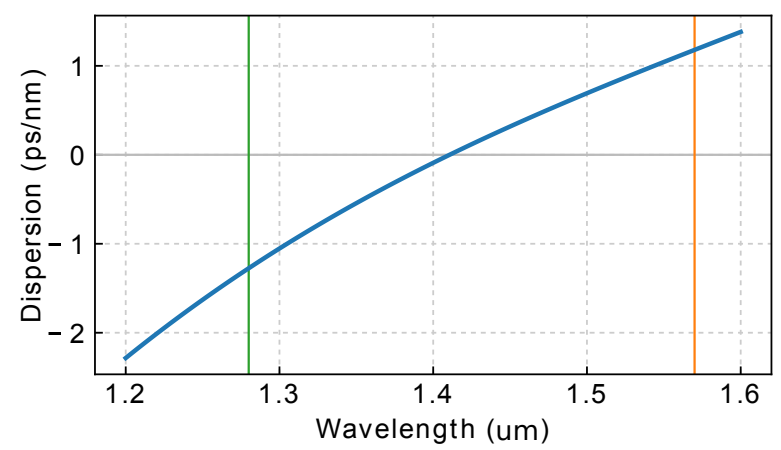

(d)

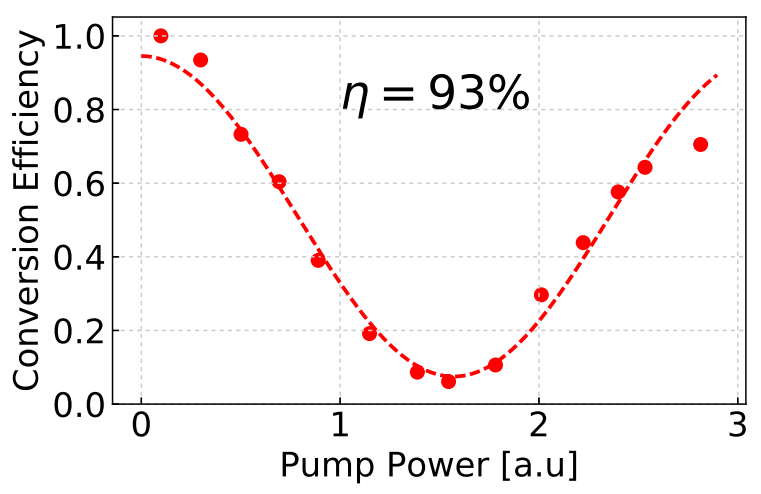

(c)

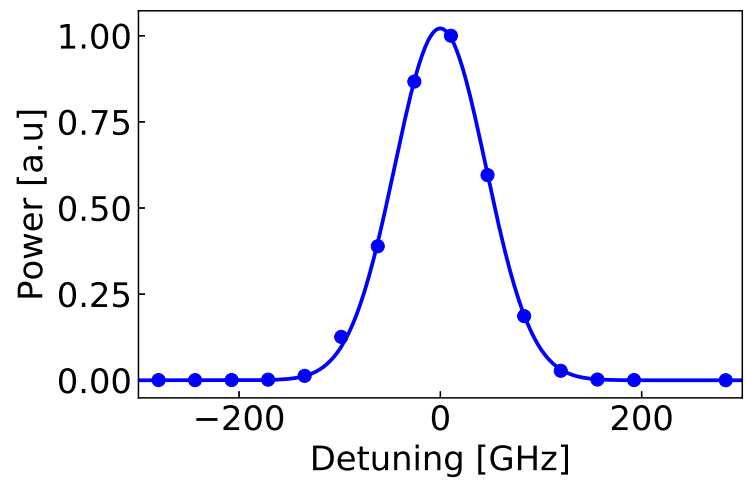

(e)

Figure S1. a) BS-FWM: Two strong pumps $\omega_{P 1}$ and $\omega_{P 2}$ drive the interaction between two fields $\omega_{s}$ and $\omega_{i}$, where the separation $\Delta \omega=\omega_{P 1}-\omega_{P 2}$ determines the frequency shift between the signal and idler fields. Phase matching is achieved by symmetric placement of the pumps, and the signal, idler fields about the ZDW of the interaction medium. b) Bragg scattering conversion efficiency as function of interaction strength $2 \gamma P$, assuming perfect phase matching. Complete conversion is achieved when the interaction strength equals $\pi / 2$. c) Measured conversion efficiency via depletion of the signal. The conversion efficiency is measured to be $93 \%$, limited by fluctuations in the pump power. d) Dispersion profile of the dispersion shifted fiber used in our implementation of BS-FWM. e) Measured bandwidth of the filtering setup centered at the target frequency. 


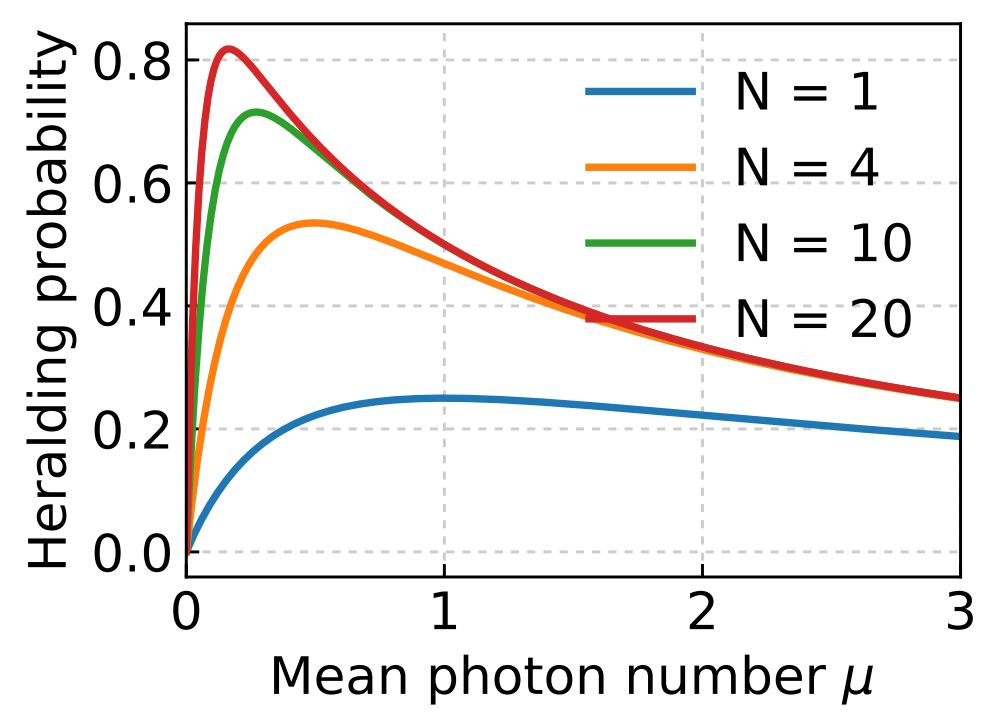

Figure S2. Heralding probability for $N$ multiplexed sources as a function of the mean photon number $\mu$ in the signal and idler modes, for a lossless switching network. Optimal $\mu$ reduces as $N$ increases.

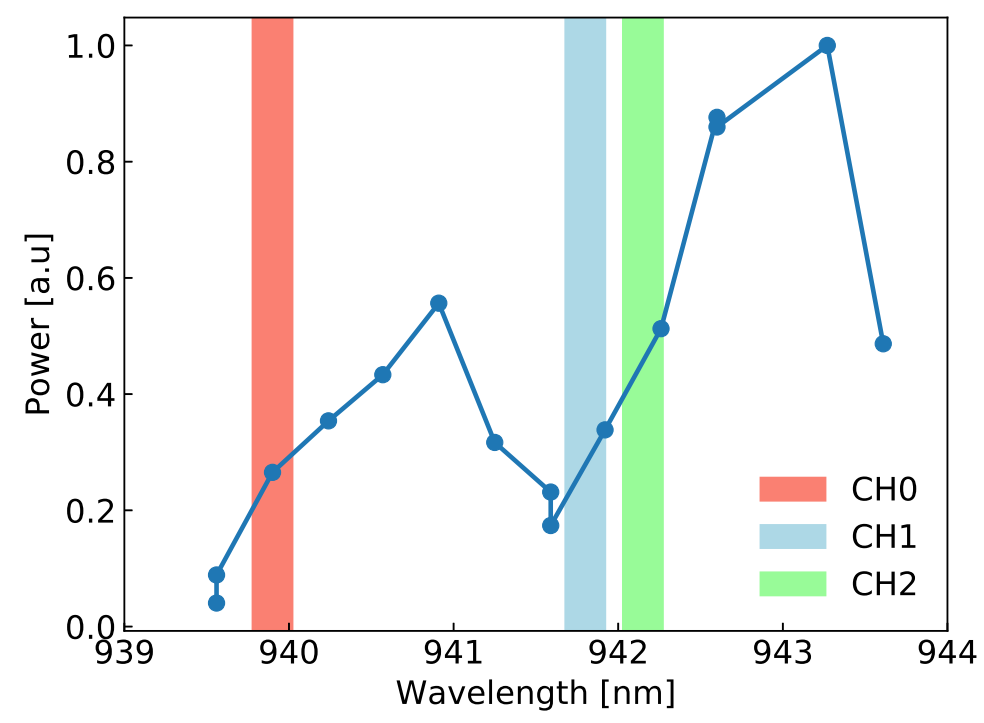

Figure S3. Spectrum of the heralding photons measured using a single photon spectrometer. The heralding photons are filtered into $100 \mathrm{GHz}$ wide channels using reflecting Bragg gratings. The highlighted regions reflect the corresponding heralded channels: $\mathrm{CH} 0, \mathrm{CH} 1, \mathrm{CH} 2$. 


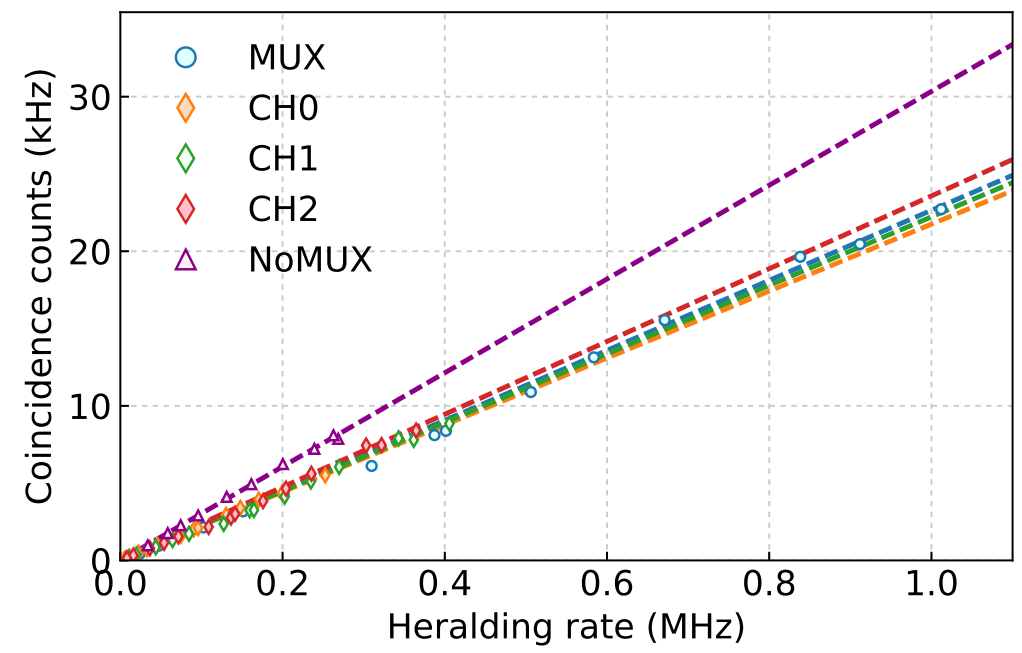

Figure S4. Characterization of heralding efficiency. The efficiency is given by the slope of the heralding rate vs heralded photon rate (measured as coincidence counts). We measure a heralding efficiency of $2.3 \%$ with the multiplexing setup in place and an efficiency of $3 \%$ without. This corresponds with the estimated $1.3 \mathrm{~dB}$ transmission loss through the BS-FWM setup measured using a classical input. 


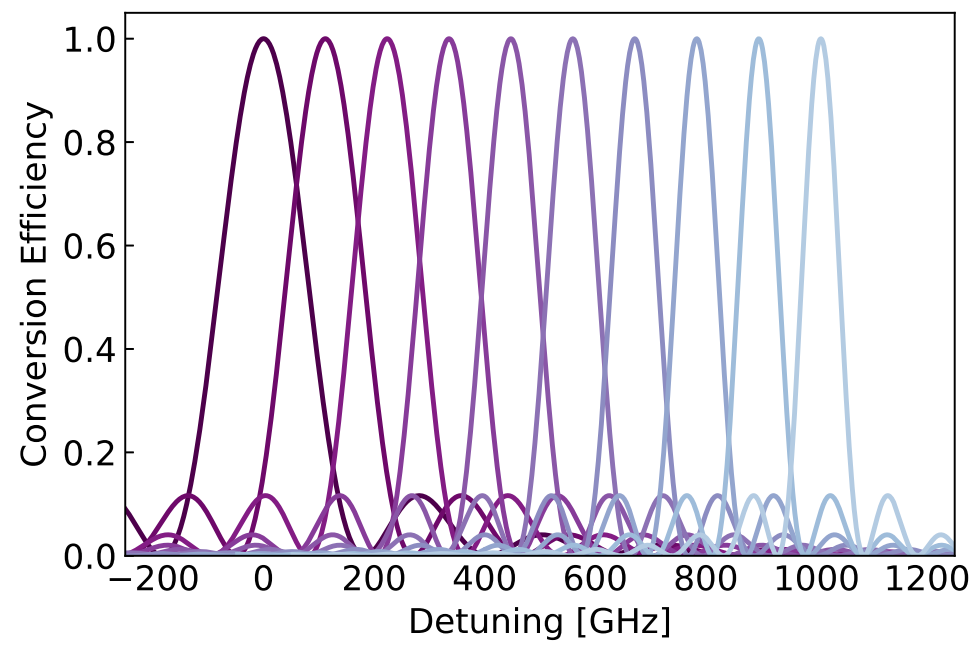

(a)

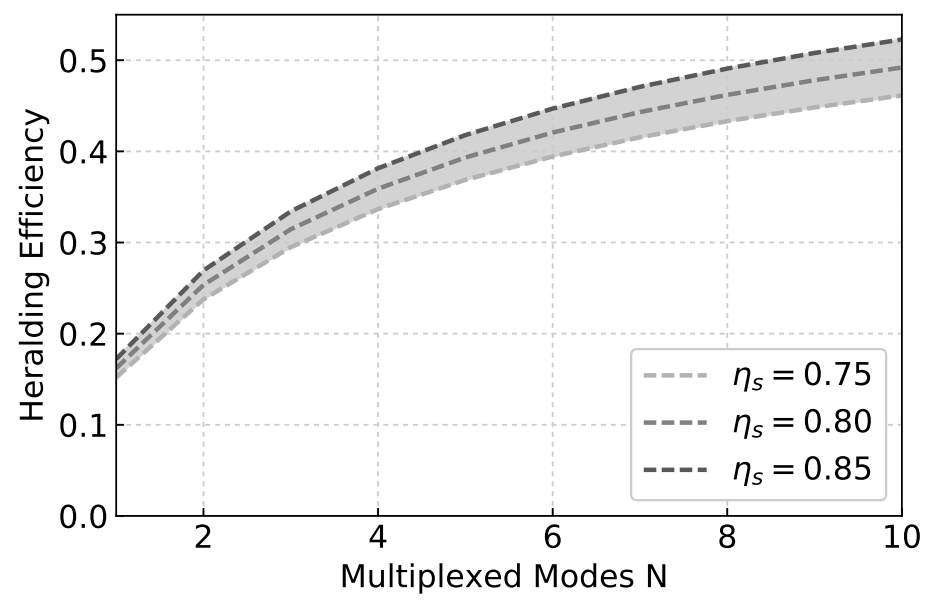

(b)

Figure S5. a) BS-FWM conversion efficiency for 10 channels separated by $100 \mathrm{GHz}$. We fix one of the two pumps while detuning the other such that the frequency separation between the two pumps matches the frequency separation between the input and the target. The conversion efficiency is maintained throughout while the acceptance bandwidth is reduced by a factor of 2 due to the effects of higher order dispersion. b) Scaling performance of frequency for 10 frequency modes, assuming a combined detection and fiber-collection efficiency of 90\%, for varying multiplexing system efficiencies $\left(\eta_{s}=0.75,0.80,0.85\right)$. Single-photon heralding efficiencies as high as $50 \%$ can be achieved with just 10 frequency modes. 